\title{
On a Fractional SPDE Driven by Fractional Noise and a Pure Jump Lévy Noise in $\mathbb{R}^{d}$
}

\author{
Xichao Sun, ${ }^{1}$ Zhi Wang, ${ }^{2}$ and Jing $\mathrm{Cui}^{3}$ \\ ${ }^{1}$ Department of Mathematics and Physics, Bengbu College, 1866 Caoshan Road, Bengbu, Anhui 233030 China \\ ${ }^{2}$ Department of Mathematics, Donghua University, 2999 North Renmin Road, Songjiang, Shanghai 201620, China \\ ${ }^{3}$ Department of Mathematics, Anhui Normal University, 1 East Beijing Road, Wuhu 241000, China
}

Correspondence should be addressed to Jing Cui; jcui123@126.com

Received 12 December 2013; Accepted 8 April 2014; Published 5 May 2014

Academic Editor: Yiming Ding

Copyright (c) 2014 Xichao Sun et al. This is an open access article distributed under the Creative Commons Attribution License, which permits unrestricted use, distribution, and reproduction in any medium, provided the original work is properly cited.

We study a stochastic partial differential equation in the whole space $x \in \mathbb{R}^{d}$, with arbitrary dimension $d \geq 1$, driven by fractional noise and a pure jump Lévy space-time white noise. Our equation involves a fractional derivative operator. Under some suitable assumptions, we establish the existence and uniqueness of the global mild solution via fixed point principle.

\section{Introduction}

Let $d \geq 1, \alpha=\left(\alpha_{1}, \ldots, \alpha_{d}\right), \delta=\left(\delta_{1}, \ldots, \delta_{d}\right)$, and $\mathbb{D}_{\delta}^{\alpha}$ be the nonlocal fractional differential operator defined by

$$
\mathbb{D}_{\delta}^{\alpha}=\sum_{i=1}^{d} D_{\delta_{i}}^{\alpha_{i}}
$$

where $D_{\delta_{i}}^{\alpha_{i}}$ denotes the fractional differential derivative with respect to the $i$ th coordinate defined via its Fourier transform $\mathscr{F}$ by

$$
\mathscr{F}\left(D_{\delta}^{\alpha} \varphi\right)(\xi)=-|\xi|^{\alpha} \exp \left(-i \delta \frac{\pi}{2} \operatorname{sgn}(\xi)\right) \mathscr{F}(\varphi)(\xi) .
$$

In this paper, we are concerned with the following jump type stochastic partial differential equation (SPDE for abbreviation) with fractional noise:

$$
\begin{aligned}
\frac{\partial u}{\partial t}(t, x)= & \mathbb{D}_{\delta}^{\alpha} u(t, x)+b(t, x, u(t, x)) \\
& +\sigma(t, x, u(t, x)) \dot{L}(t, x)+B^{H}(d t, d x), \\
u(0, x)= & u_{0}(x),
\end{aligned}
$$

where $(t, x) \in[0, T] \times \mathbb{R}^{d}, \dot{L}$ is a pure jump Lévy spacetime white noise on $[0, T] \times \mathbb{R}^{d}$ defined on a complete probability space $(\Omega, \mathscr{F}, P)$, and $B^{H}(d t, d x)$ denotes the fractional noise on $[0, T] \times \mathbb{R}^{d}$ with multiparameter $H=$ $\left(h_{0}, h_{1}, \ldots, h_{d}\right)$ for $h_{i} \in(0,1)$ and $i \in\{0,1,2, \ldots, d\}$ defined on a complete probability space $(\Omega, \mathscr{F}, P)$ (see Section 2 for precise definitions). Actually, we understand this equation as Walsh [1] sense, and so we can rewrite (3) as follows:

$$
\begin{aligned}
& u(t, x) \\
& =\int_{\mathbb{R}^{d}} \mathbb{G}_{\alpha, \delta}(t, x-y) u_{0}(y) d y \\
& \quad+\int_{0}^{t} \int_{\mathbb{R}^{d}} \mathbb{G}_{\alpha, \delta}(t-s, x-y) B^{H}(d y, d s) \\
& \quad+\int_{0}^{t} \int_{\mathbb{R}^{d}} \mathbb{G}_{\alpha, \delta}(t-s, x-y) b(s, y, u(s, y)) d y d s \\
& \quad+\int_{0}^{t} \int_{\mathbb{R}^{d}} \mathbb{G}_{\alpha, \delta}(t-s, x-y) \sigma(s, y, u(s, y)) \dot{L}(y, s) d y d s,
\end{aligned}
$$

for all $t \in[0, T]$ and $x \in \mathbb{R}^{d}$, where $\mathbb{G}_{\alpha, \delta}(\cdot, *)$ denotes the Green function associated with (3).

In the present paper, we are interested in the study of (3) with respect to $d$-dimensional nonlocal fractional differential operator $\mathbb{D}_{\delta}^{\alpha}$. Such operator is initially introduced by Debbi 
and Dozzi [2] for $d=1$ and is generalized by Boulanba et al. [3] for the multidimensional space $\mathbb{R}^{d}$. This operator is a generalization of various well-known operators, such as the Laplacian operator, the inverse of the generalized Riesz-Feller potential, and the Riemann-Liouville differential operator. In probabilistic terms, replacing the Laplacian by its fractional power (which is an integrodifferential operator) leads to interesting and largely open questions of extensions of results for Brownian motion driven stochastic equations to those driven by Lévy stable processes. In the physical literature, such fractal anomalous diffusions have been recently enthusiastically embraced by a slew of investigators in the context of hydrodynamics, acoustics, trapping effects in surface diffusion, statistical mechanics, relaxation phenomena, and biology. We refer the readers to [4-9] and references therein for more information about such fractional differential operator.

On the other hand, many researchers are interested in studying SPDES driven by a fractional noise. The heat equations with a multiparameter fractional noise of Hurst parameter $H=\left(h_{0}, \ldots, h_{d}\right)$ on $[0, \infty] \times \mathbb{R}^{d}$ were introduced by $\mathrm{Hu}[10]$, and he showed the existence and uniqueness of the solutions to the equation, via chaos expansion. Wei [11] considered a class of four-order stochastic partial differential equations driven by the multiparameter fractional noise; the author studied the regularity of the solution and the existence of the density of the law of the solution. Hu and Nualart [12] studied the $d$-dimensional stochastic heat equation with a multiplicative Gaussian noise which is white in space and has the covariance of a fractional Brownian motion with Hurst parameter $h \in(0,1)$ in time. More works for the fields can be found in Balan and Tudor [13, 14], Bo et al. [15, 16], Jiang et al. [17-19], and Shi and Wang [20] and the references therein. In the meanwhile, there also have been some works on SPDES involving Lévy space-time white noise (e.g., Albeverio et al. [21], Shi and Wang [22], Truman and $\mathrm{Wu}[23,24]$, and $\mathrm{Wu}$ and Xie [25]). Løkka et al. [26] studied the stochastic partial differential equations driven by a $d$-parameter pure jump Lévy white noise. As an example they used this theory to solve the stochastic Poisson equation with respect to Lévy white noise for any dimension $d$. We notice that the fixed point principle and Picard iteration scheme work in Albeverio et al. [21] and Truman and Wu [23, 24], since BurkhölderDavis-Gundy (B-D-G) inequality can be applied to estimate stochastic integral with respect to compensated Poisson random measure in $L^{2}$-sense. Unfortunately, the usual B-D-G inequality cannot work in estimating stochastic integral with respect to compensated Poisson random measure in $L^{p}$-sense $(p>2)$. Hence a new version of B-D-G inequality will be adopted for $L^{p}(p \geq 2)$-estimates on the solution to (3) (see Bo and Wang [27]).

Motivated by the above results, in this paper, we study a stochastic fractional partial differential equation in the whole space $x \in \mathbb{R}^{d}$, with arbitrary dimension $d \geq 1$, driven by fractional noise and a pure jump Lévy space-time white noise. The main subject of this paper is to establish the existence and uniqueness of the solution of (3) via fixed point principle.
The rest of the paper is organized as follows. In Section 2, we begin by making some notations and by recalling some basic preliminaries about fractional noises, Lévy space-time white noise, and Green function which will be needed later. In Section 3, we will prove the existence and uniqueness of the mild solution to (4) in $L^{p}(p \geq 2)$ sense under some approximate conditions. Most of the estimates of this paper contain unspecified constants. An unspecified positive and finite constant will be denoted by $C$, which may not be the same in each occurrence. Sometimes we will emphasize the dependence of these constants upon parameters.

\section{Preliminaries}

In this section, we will present the definitions and some results of the multiparameter fractional noises, Lévy spacetime white noise, and Green function.

2.1. Multiparameter Fractional Noises. Recall that a fractional Brownian motion with Hurst parameter $h \in(0,1)$ on $[0, T]$ is a centered Gaussian process $\left(B_{t}\right)$ with covariance

$$
E\left(B_{t} B_{s}\right)=\frac{1}{2}\left(t^{2 h}+s^{2 h}-|t-s|^{2 h}\right) .
$$

Following Hu [10] and Wei [11], we introduce a multiparameter fractional Brownian field.

Definition 1. A multiparameter fractional Brownian field $B^{H}=\left\{B^{H}(t, x),(t, x) \in[0, T] \times \mathbb{R}^{d}\right\}$ with multiparameter $H=\left(h_{0}, h_{1}, \ldots, h_{d}\right)$ for $h_{i} \in(0,1)$ and $i \in\{0,1,2, \ldots, d\}$ is a centered Gaussian field defined on some probability space $(\Omega, \mathscr{F}, P)$ with covariance

$$
\begin{aligned}
& E\left[B^{H}(t, x) B^{H}(s, y)\right] \\
& =\frac{1}{2^{d+1}}\left(t^{2 h_{0}}+s^{2 h_{0}}-|t-s|^{2 h_{0}}\right) \\
& \quad \times \prod_{i=1}^{d}\left(x_{i}^{2 h_{i}}+y_{i}^{2 h_{i}}-\left|x_{i}-y_{i}\right|^{2 h_{i}}\right),
\end{aligned}
$$

for all $t, s \in[0, T]$ and $x=\left(x_{1}, \ldots, x_{d}\right), y=\left(y_{1}, \ldots, y_{d}\right) \in$ $\mathbb{R}^{d}$.

Throughout the paper, we limit our consideration on the multiparameter fractional Brownian field with parameter $H=\left(h_{0}, h_{1}, \ldots, h_{d}\right)=(h, h, \ldots, h)$ and $h \in(1 / 2,1)$.

Let

$$
\begin{array}{r}
\Psi_{h}(t, s, x, y):=[h(2 h+1)]^{d+1}|t-s|^{2 h-2} \prod_{i=1}^{d}\left|x_{i}-y_{i}\right|^{2 h-2}, \\
0 \leq s \leq T, x, y \in \mathbb{R}^{d} .
\end{array}
$$


Introduce the following Hilbert space:

$$
\begin{aligned}
L_{\Psi}^{2}:=\left\{f:[0, T] \times \mathbb{R}^{d} \longrightarrow\right. & \mathbb{R} ; \\
\|f\|_{\Psi}^{2}=\int_{[0, T]^{2}} \int_{\mathbb{R}^{2 d}} \Psi_{h}(t, s, x, y) & \\
& \times f(t, x) f(s, y) d y d x d s d t<\infty\},
\end{aligned}
$$

where $d x=d x_{1} d x_{2}, \ldots, d x_{d}$ and $d y=d y_{1} d y_{2}, \ldots, d y_{d}$. Let $f \in L_{\Psi}^{2}$; one can define the following stochastic integral:

$$
\left\{\int_{0}^{t} \int_{\mathbb{R}^{d}} f(s, x) d B^{H}(t, x) ; t \in[0, T]\right\},
$$

(see, e.g., $\mathrm{Hu}[10]$ ); it is easy to check that this integral has the usual properties as a stochastic integral.

Proposition 2. Let $f, g \in L_{\Psi}^{2}$. Then

(1) $E\left[\int_{0}^{T} \int_{\mathbb{R}^{d}} f(s, x) d B^{H}(s, x)\right]=0$,

(2) $E\left[\int_{0}^{T} \int_{\mathbb{R}^{d}} f(s, x) d B^{H}(s, x) \int_{0}^{T} \int_{\mathbb{R}^{d}} g(s, x) d B^{H}(s, x)\right]=$ $\langle f, g\rangle_{L_{\Psi}^{2}}$

The following embedding proposition which is an extension to the results in Mémin et al. [28] had been proved in Wei [11].

Lemma 3. Consider the following:

$L^{h}\left([0, T] \times \mathbb{R}^{d}\right) \subset L_{\Psi}^{2}(h>1 / 2)$.

2.2. Lévy Space-Time White Noise. Let $\left(E_{i}, \mathscr{E}_{i}, \mu_{i}\right), i=1,2$, be two $\sigma$-finite measurable spaces. We call $N:\left(E_{1}, \mathscr{E}_{1}, \mu_{1}\right) \times$ $\left(E_{2}, \mathscr{E}_{2}, \mu_{2}\right) \times(\Omega, \mathscr{F}, P) \rightarrow \mathbb{N} \cup\{0\} \cup\{\infty\}$ a Poisson noise on $\left(E_{1}, \mathscr{E}_{1}, \mu_{1}\right)$, if, for all $A \in \mathscr{E}_{1}, B \in \mathscr{E}_{2}$, and $n \in \mathbb{N} \cup\{0\} \cup\{\infty\}$,

$$
P(N(A, B)=n)=\frac{e^{-\mu_{1}(A) \mu_{2}(B)\left[\mu_{1}(A) \mu_{2}(B)\right]^{n}}}{n !} .
$$

In particular, if $\left(E_{1}, \mathscr{E}_{1}, \mu_{1}\right)=\left([0, \infty) \times \mathbb{R}^{d}, \mathscr{B}([0, \infty) \times\right.$ $\left.\left.\mathbb{R}^{d}\right), d t \times d x\right)$, then define a compensated random martingale measure by

$$
M(B, A, t)=N([0, t] \times A, B)-\mu_{1}([0, t] \times A) \mu_{2}(B),
$$

by assuming that $\mu_{1}([0, t] \times A) \mu_{2}(B)<\infty$ for all $(t, A, B) \epsilon$ $[0, \infty) \times \mathscr{B}\left(\mathbb{R}^{d}\right) \times \mathscr{E}_{2}$. Further, let $\phi: E_{1} \times E_{2} \times \Omega \rightarrow \mathbb{R}$ be an $\left(\mathscr{F}_{t}\right)_{t \geq 0}$-predictable function satisfying

$$
\mathbb{E}\left[\int_{0}^{t} \int_{A} \int_{B}|\phi(t, x, y)|^{2} \mu_{2}(d y) d x d s\right]<\infty,
$$

for all $t>0$ and $(A, B) \in \mathscr{E}_{1} \times \mathscr{E}_{2}$. We can define a stochastic integral process

$$
\left(R_{t}=\int_{0}^{t+} \int_{A} \int_{B} \phi(s, x, y) M(d y, d x, d s)\right)
$$

which is a square integrable $\left(P,\left(\mathscr{F}_{t}\right)_{t \geq 0}\right)$-martingale. It is well known that a (pure jump) Lévy space-time white noise possesses the following structure:

$$
\begin{aligned}
\dot{L}(x, t)= & \int_{U_{0}} h_{1}(t, x, y) \dot{M}(d y, x, t) \\
& +\int_{E_{2} \backslash U_{0}} h_{2}(t, x, y) \dot{N}(d y, x, t),
\end{aligned}
$$

for some $U_{0} \in E_{2}$ such that $\mu_{2}\left(E_{2} \backslash U_{0}\right)<\infty$ and $\int_{U_{0}} z^{2} \mu_{2}(d z)<+\infty$. Here $h_{1}, h_{2}:[0, \infty) \times \mathbb{R} \times E_{2} \rightarrow \mathbb{R}$ are some measurable functions; $\dot{M}$ and $\dot{N}$ are the RadonNikodym derivatives given by

$$
\begin{aligned}
& \dot{M}(d y, x, t)=\frac{M(d y, d x, d t)}{d t \times d x}, \\
& \dot{N}(d y, x, t)=\frac{N(d t \times d x, d y)}{d t \times d x},
\end{aligned}
$$

with $(t, x, y) \in[0, \infty) \times \mathbb{R}^{d} \times E_{2}$.

The following B-D-G inequality is given by Bo and Wang [27], which is useful to estimate the higher order moments of mild solution to (4).

Lemma 4. Let $\phi:[0, \infty) \times \mathbb{R}^{d} \times E_{2} \times \Omega \rightarrow \mathbb{R}$ be $\left(\mathscr{F}_{t}\right)_{t \geq 0^{-}}$ predictable and satisfy (13). Define the integral process by

$$
\left\{X_{t}=\int_{0}^{t+} \int_{\mathbb{R}^{d}} \int_{E_{2}} \phi(s, y, z) M(d z, d y, d s), t \geq 0\right\} .
$$

Then, for any $T>0$ and $p>1$, there exists a constant $C_{p, T}>0$ such that

$$
\begin{aligned}
& \sup _{t \in[0, T]} \mathbb{E}\left[\left|X_{t}\right|^{p}\right] \\
& \leq C_{p, T}\left[\int_{0}^{T} \int_{\mathbb{R}^{d}} \int_{E_{2}}\left(\mathbb{E}\left[|\phi(s, y, z)|^{p}\right]\right)^{2 / p} \mu_{2}(d z) d y d s\right]^{p / 2} .
\end{aligned}
$$

2.3. Green Function. In this subsection, we will introduce the nonlocal fractional differential operator defined by

$$
\mathbb{D}_{\delta}^{\alpha}=\sum_{i=1}^{d} D_{\delta_{i}}^{\alpha_{i}}
$$

where $\alpha=\left(\alpha_{1}, \ldots, \alpha_{d}\right), \delta=\left(\delta_{1}, \ldots, \delta_{d}\right)$, and $D_{\delta_{i}}^{\alpha_{i}}$ denotes the fractional differential derivative with respect to the $i$ th coordinate defined via its Fourier transform $\mathscr{F}$ by

$$
\mathscr{F}\left(D_{\delta}^{\alpha} \varphi\right)(\xi)=-|\xi|^{\alpha} \exp \left(-i \delta \frac{\pi}{2} \operatorname{sgn}(\xi)\right) \mathscr{F}(\varphi)(\xi) .
$$

In this paper, we will assume that $\left|\delta_{i}\right| \leq \min \left\{\alpha_{i}-\left[\alpha_{i}\right]_{2}, 2+\right.$ $\left.\left[\alpha_{i}\right]_{2}-\alpha_{i}\right\}, i=1, \ldots, d,\left[\alpha_{i}\right]_{2}$ is the largest even integer less than or equal to $\alpha_{i}$ (even part of $\alpha_{i}$ ), and $\delta_{i} \in 2 \mathbb{N}+1$. 
In one space dimension, the operator $D_{\delta}^{\alpha}$ is a closed, densely defined operator on $L^{2}(\mathbb{R})$ and it is the infinitesimal generator of a semigroup which is in general not symmetric and not a contraction. This operator is a generalization of various well-known operators, such as the Laplacian operator (when $\alpha=2$ ), the inverse of the generalized RieszFeller potential (when $\alpha>2$ ), and the Riemann-Liouville differential operator (when $|\delta|=2+[\alpha]_{2}$ or $|\delta|=\alpha-[\alpha]$ ). It is self-adjoint only when $\delta=0$ and, in this case, it coincides with the fractional power of the Laplacian. We refer the readers to Debbi [29], Debbi and Dozzi [2], and Komatsu [30] for more details about this operator.

According to Komatsu [30], $D_{\delta}^{\alpha}$ can be represented, for $1<\alpha<2$, by

$$
\begin{aligned}
& D_{\delta}^{\alpha} \varphi(x) \\
& \quad=\int_{\mathbb{R}} \frac{\varphi(x+y)-\varphi(x)-y \varphi^{\prime}(x)}{|y|^{1+\alpha}} \\
& \quad \times\left(\kappa_{-}^{\delta} 1_{(-\infty, 0)}(y)+\kappa_{+}^{\delta} 1_{(-0,+\infty)}(y)\right) d y
\end{aligned}
$$

and, for $0<\alpha<1$, by

$$
\begin{aligned}
& D_{\delta}^{\alpha} \varphi(x) \\
& \quad=\int_{\mathbb{R}} \frac{\varphi(x+y)-\varphi(x)}{|y|^{1+\alpha}} \\
& \quad \times\left(\kappa_{-}^{\delta} 1_{(-\infty, 0)}(y)+\kappa_{+}^{\delta} 1_{(-0,+\infty)}(y)\right) d y,
\end{aligned}
$$

where $\kappa_{-}^{\delta}$ and $\kappa_{+}^{\delta}$ are two nonnegative constants satisfying $\kappa_{-}^{\delta}+$ $\kappa_{+}^{\delta}>0$ and $\varphi$ is a smooth function for which the integral exists, and $\varphi^{\prime}$ is its derivative. This representation identifies it as the infinitesimal generator for a nonsymmetric $\alpha$-stable Lévy process.

Let $G_{\alpha, \delta}(t, x)$ be the fundamental solution of the following Cauchy problem:

$$
\begin{gathered}
\frac{\partial u}{\partial t}(t, x)=D_{\delta}^{\alpha}(t, x), \\
u(0, x)=\delta_{0}(x), \quad t>0, x \in \mathbb{R},
\end{gathered}
$$

where $\delta_{0}(\cdot)$ is the Dirac distribution. By Fourier transform, we see that $D_{\delta}^{\alpha}(t, x)$ is given by $\mathbb{F}$ :

$$
\begin{aligned}
& G_{\alpha, \delta}(t, x) \\
& =\frac{1}{2 \pi} \int_{\mathbb{R}} \exp \left(-i z x-t|z|^{\alpha} \exp \left(-i \delta \frac{\pi}{2} \operatorname{sgn}(z)\right)\right) d z .
\end{aligned}
$$

The relevant parameters $\alpha$ called the index of stability and $\delta$ (related to the asymmetry) improperly referred to as the skewness are real numbers satisfying $|\delta| \leq \min \left\{\alpha-[\alpha]_{2}, 2+\right.$ $\left.[\alpha]_{2}-\alpha\right\}$, and $\delta=0$ when $\delta \in 2 \mathbb{N}+1$.

Let us list some known facts on $G_{\alpha, \delta}(t, x)$ which will be used later on (see, e.g., Debbi [29] and Debbi and Dozzi [2]).
Lemma 5. Let $\alpha \in(0, \infty) /\{\mathbb{N}\}$; one has the following.

(1) The function $G_{\alpha, \delta}(t, \cdot)$ is not in general symmetric relatively to $x$ and it is not everywhere positive.

(2) For any $s, t \in(0, \infty)$ and $x \in \mathbb{R}$,

$$
\frac{\partial^{n}}{\partial x^{n}} G_{\alpha, \delta}(t, x)=(s)^{-(n+1) / \alpha} G_{\alpha, \delta}\left(s^{-1} t, s^{-1 / \alpha} x\right),
$$

or equivalently

$$
\frac{\partial^{n}}{\partial x^{n}} G_{\alpha, \delta}(t, x)=(t)^{-(n+1) / \alpha} G_{\alpha, \delta}\left(1,(t)^{-1 / \alpha} x\right) .
$$

(3) $G_{\alpha}(s, \cdot) * G_{\alpha, \delta}(t, \cdot)=G_{\alpha, \delta}(s+t, \cdot)$ for any $s, t \in(0, \infty)$.

(4) For $n \geq 1$, there exist some constants $C$ and $C_{n}>0$ such that, for all $x \in \mathbb{R}$,

$$
\begin{gathered}
\left|G_{\alpha, \delta}(1, x)\right| \leq C \frac{1}{1+|x|^{1+\alpha}}, \\
\left|\frac{\partial^{n}}{\partial x^{n}} G_{\alpha, \delta}(1, x)\right| \leq C_{n} \frac{|x|^{\alpha+n-1}}{\left(1+|x|^{\alpha+n}\right)^{2}} .
\end{gathered}
$$

(5) $\int_{0}^{T} \int_{\mathbb{R}}\left|G_{\alpha, \delta}(t, x)\right|^{\lambda} d t d x<\infty$ if and only if $1 / \alpha<\lambda$

For $d \geq 1$ and any multi-index $\alpha=\left(\alpha_{1}, \ldots, \alpha_{d}\right)$ and $\delta=\left(\delta_{1}, \ldots, \delta_{d}\right)$, let $\mathbb{G}_{\alpha, \delta}(t, x)$ be the Green function of the deterministic equation

$$
\begin{aligned}
\frac{\partial u}{\partial t}(t, x) & =\mathbb{D}_{\delta}^{\alpha}(t, x), \\
u(0, x) & =\delta_{0}(x), \quad t>0, x \in \mathbb{R}^{d} .
\end{aligned}
$$

Clearly

$$
\begin{aligned}
& \mathbb{G}_{\alpha, \delta}(t, x) \\
& =\prod_{i=1}^{d} G_{\alpha_{i}, \delta_{i}}\left(t, x_{i}\right) \\
& =\frac{1}{(2 \pi)^{d}} \\
& \quad \times \int_{\mathbb{R}^{d}} \exp \left(-i\langle\xi, x\rangle-t \sum_{i=1}^{d}\left|\xi_{i}\right|^{\alpha_{i}} \exp \left(-i \delta \frac{\pi}{2} \operatorname{sgn}\left(\xi_{i}\right)\right)\right) d \xi .
\end{aligned}
$$




\section{Existence and Uniqueness}

In this section, we are going to prove the existence and uniqueness of the global mild solution to (3). Recall (4) and (14). Then, for all $(t, x) \in[0, T] \times \mathbb{R}^{d}$,

$$
\begin{aligned}
& u(t, x) \\
& =\int_{\mathbb{R}^{d}} \mathbb{G}_{\alpha, \delta}(t, x-y) u_{0}(y) d y \\
& \quad+\int_{0}^{t} \int_{\mathbb{R}^{d}} \mathbb{G}_{\alpha, \delta}(t-s, x-y) B^{H}(d y, d s) \\
& +\int_{0}^{t} \int_{\mathbb{R}^{d}} \mathbb{G}_{\alpha, \delta}(t-s, x-y) b(s, y, u(s, y)) d y d s \\
& \quad+\int_{0}^{t} \int_{\mathbb{R}^{d}} \mathbb{G}_{\alpha, \delta}(t-s, x-y) \sigma(s, y, u(s, y)) \psi(s, y) d y d s \\
& \quad+\int_{0}^{t+} \int_{\mathbb{R}^{d}} \int_{E_{2}} \mathbb{G}_{\alpha, \delta}(t-s, x-y) \\
& \times \sigma(s, y, u(s, y)) h(s, y, z) M(d z, d y, d s),
\end{aligned}
$$

with the mappings

$$
\begin{gathered}
\psi(t, y)=\int_{E_{2} \backslash U_{0}} h_{2}(t, y, z) \mu_{2}(d z), \\
h(t, y, z)=h_{1}(t, y, z) 1_{U_{0}}(z)+h_{2}(t, y, z) 1_{E_{2} \backslash U_{0}}(z),
\end{gathered}
$$

with indicator $1_{A}(\cdot)$ of the set $A \in \mathscr{E}_{2}$.

In the following, we will show that such a mild solution indeed exists and is unique, which is stated as follows.

Theorem 6. Let $1+\sum_{i=1}^{d}\left(1 / \alpha_{i}-1 / h \alpha_{i}\right)>0$. Suppose the following conditions hold.

(1) b, $\sigma$ are uniformly Lipschitzian; that is, there exists a constant $C>0$ such that for $(t, x) \in[0, T] \times \mathbb{R}^{d}$ and $u, v \in \mathbb{R}$

$|b(t, x, u)-b(t, x, v)|+|\sigma(t, x, u)-\sigma(t, x, v)| \leq C|u-v|$

(2) $b$ is linear growth; that is, there exists a constant $C>0$ such that for $u \in \mathbb{R}$

$$
|b(t, x, u)| \leq C(1+|u|) .
$$

(3) For $p \in\left(2\left(1+\sum_{i=1}^{d}\left(1 / \alpha_{i}\right)\right) /\left(\sum_{i=1}^{d}\left(1 / \alpha_{i}\right)-1\right),+\infty\right)$ with $\alpha>1$,

$$
\begin{gathered}
\sup _{t \in[0, T]}\|\psi(t, \cdot)\|_{p}^{p}<\infty, \\
\sup _{t \in[0, T]}\left\|\int_{E_{2}}|h(t, \cdot, z)|^{2} \mu_{2}(d z)\right\|_{p / 2}^{p / 2}<\infty .
\end{gathered}
$$

Then, for all $\mathscr{F}_{0}$-measurable $u_{0}: \mathbb{R}^{d} \times \Omega \rightarrow \mathbb{R}$ satisfying $\mathbb{E}\left[\left\|u_{0}(\cdot)\right\|_{p}^{p}\right]<\infty$, there exists a unique mild solution $(u(t, x))_{(t, x) \in[0, T] \times \mathbb{R}^{d}}$ to (3) and, for all $p \in$ $\left(2\left(1+\sum_{i=1}^{d}\left(1 / \alpha_{i}\right)\right) /\left(\sum_{i=1}^{d}\left(1 / \alpha_{i}\right)-1\right),+\infty\right)$,

$$
\sup _{t \in[0, T]} \mathbb{E}\left[\|u(t, \cdot)\|_{p}^{p}\right]<\infty
$$

In order to prove the above theorem, we need the following lemmas.

Lemma 7. Suppose $p \in[1, \infty), \rho \in[1, p]$, and $r \in[1, \infty)$ such that

$$
\frac{1}{r}=\frac{1}{p}-\frac{1}{\rho}+1 \in[0,1] .
$$

Let $\mathbb{G}_{\alpha, \delta}=\mathbb{G}_{\alpha, \delta}(t, x-y)$ be the Green kernel, $H=\mathbb{G}_{\alpha, \delta}$, or $\mathbb{G}_{\alpha, \delta}^{2}$ with $(t, x, y) \in[0, T] \times \mathbb{R}^{d} \times \mathbb{R}^{d}$. Define an operator $\mathscr{A}$ by

$$
\mathscr{A}(v)(t, x)=\int_{0}^{t} \int_{\mathbb{R}^{d}} H(t-s, x-y) v(s, y) d y d s,
$$

with $v \in L^{1}\left([0, T] ; L^{\rho}()\right)$. Then $\mathscr{A}: L^{1}\left([0, T] ; L^{\rho}()\right) \rightarrow$ $L^{\infty}\left([0, T] ; L^{\rho}()\right)$ is bounded linear operator and satisfies the following.

(a) If $H=\mathbb{G}_{\alpha, \delta}$, then there exists a constant $C>0$ such that for all $r \in\left[1,1+\min \alpha_{i}\right)$

$$
\begin{aligned}
& \|\mathscr{A}(v)(t, \cdot)\|_{p} \\
& \leq C \int_{0}^{t}(t-s)^{\sum_{i=1}^{d}\left((r-1) / \alpha_{i}\right)}\|v(x, \cdot)\|_{\rho} d s, \quad \forall t \in[0, T] .
\end{aligned}
$$

(b) If $\mathbb{G}_{\alpha, \delta}^{2}$, then there exists a constant $C>0$ such that for all $r \in\left[1,\left(1+\min \alpha_{i}\right) / 2\right)$

$$
\begin{aligned}
& \|\mathscr{A}(v)(t, \cdot)\|_{p} \\
& \leq C \int_{0}^{t}(t-s)^{\sum_{i=1}^{d}\left((r-2) / \alpha_{i}\right)}\|v(x, \cdot)\|_{\rho} d s, \quad \forall t \in[0, T] .
\end{aligned}
$$

Proof. We only need to prove the case (a), since the proof of (b) is similar. Together with Minkowski's inequality, (3) of Lemma 5, and Young inequality, one can get

$$
\begin{aligned}
& \|\mathscr{A}(v)(t, \cdot)\|_{p} \\
& =\left\|\int_{0}^{t} \int_{\mathbb{R}^{d}} \mathbb{G}_{\alpha, \delta}(t-s, \cdot-y) v(s, y) d y d s\right\|_{p}
\end{aligned}
$$




$$
\begin{aligned}
& \leq \int_{0}^{t}\left\|\int_{\mathbb{R}^{d}} \mathbb{G}_{\alpha, \delta}(t-s, \cdot-y) v(s, y) d y\right\|_{p} d s \\
& \leq C \int_{0}^{t}(t-s)^{-\sum_{i=1}^{d}\left(1 / \alpha_{i}\right)} \\
& \quad \times\left\|\int_{\mathbb{R}^{d}} \prod_{i=1}^{d} G_{\alpha_{i}, \delta_{i}}\left(1,(t-s)^{-1 / \alpha_{i}}\left(\cdot-y_{i}\right)\right)|v(s, y)| d y\right\|_{p} d s \\
& \leq C \int_{0}^{t}(t-s)^{-\sum_{i=1}^{d}\left(1 / \alpha_{i}\right)} \\
& \quad \times\left\|\left(\prod_{i=1}^{d} G_{\alpha_{i}, \delta_{i}}\left(1,(t-s)^{-1 / \alpha_{i}}(\cdot)\right)\right) *|v(s, \cdot)|\right\|_{p} d s \\
& \leq C \int_{0}^{t}(t-s)^{-\sum_{i=1}^{d}\left(1 / \alpha_{i}\right)} \\
& \quad \times\left\|\prod_{i=1}^{d} G_{\alpha_{i}, \delta_{i}}\left(1,(t-s)^{-1 / \alpha_{i}}(\cdot)\right)\right\|_{r} \cdot\|v(s, \cdot)\|_{\rho} d s \\
& \leq C \int_{0}^{t}(t-s)^{-\sum_{i=1}^{d}\left(1 / \alpha_{i}\right)}\|v(s, \cdot)\|_{\rho} d s,
\end{aligned}
$$

where we have used the fact that for $r \in\left[1,1+\min \alpha_{i}\right)$

$$
\begin{aligned}
& \left\|\prod_{i=1}^{d} G_{\alpha_{i}, \delta_{i}}\left(1,(t-s)^{-1 / \alpha_{i}}(\cdot)\right)\right\|_{r} \\
& =\left[\prod_{i=1}^{d}\left(\int_{\mathbb{R}}\left|G_{\alpha_{i}, \delta_{i}}\left(1,(t-s)^{-1 / \alpha_{i}} y_{i}\right)\right|^{r} d y_{i}\right)\right]^{1 / r} \\
& =(t-s)^{\sum_{i=1}^{d}\left(r / \alpha_{i}\right)}\left[\prod_{i=1}^{d}\left(\int_{\mathbb{R}}\left|G_{\alpha_{i}, \delta_{i}}\left(1, z_{i}\right)\right|^{r} d z_{i}\right)\right]^{1 / r} \\
& \leq C(t-s)^{\sum_{i=1}^{d}\left(r / \alpha_{i}\right)} .
\end{aligned}
$$

The proof of this lemma is completed.

In the following, we mainly adopt the fixed point principle to prove Theorem 6. Let $\mathbb{B}$ be the space of all $L^{p}(\mathbb{R})$-valued $\mathscr{F}_{t}$-adapted processes $u(t, \cdot)_{0 \leq t \leq T}:[0, T] \times \mathbb{R}^{d} \times \Omega \rightarrow \mathbb{R}$ with the norm

$$
\|a\|_{\mathbb{B}}:=\left[\sup _{0 \leq t \leq T} e^{-\eta t} \mathbb{E}\left[\|u(t, \cdot)\|_{p}^{p}\right]\right]^{1 / p}, \quad \eta>0 .
$$

Then $\left(\mathbb{B},\|\cdot\|_{\mathbb{B}}\right)$ is a Banach space. Now, for $u \in \mathbb{B}$, let us define an operator $\mathscr{T}$ by

$$
\mathscr{T}(t, x)=\sum_{i=1}^{5} \mathscr{T}_{i}(u)(t, x)
$$

where

$$
\begin{aligned}
& \mathscr{T}_{1}(u)(t, x)=\int_{\mathbb{R}^{d}} \mathbb{G}_{\alpha, \delta}(t, x-y) u_{0}(y) d y, \\
& \mathscr{T}_{2}(u)(t, x)=\int_{0}^{t} \int_{\mathbb{R}^{d}} \mathbb{G}_{\alpha, \delta}(t-s, x-y) b(s, y, u(s, y)) d y d s, \\
& \mathscr{T}_{3}(u)(t, x)=\int_{0}^{t} \int_{\mathbb{R}^{d}} \mathbb{G}_{\alpha, \delta}(t-s, x-y) B^{H}(d y, d s), \\
& \mathscr{T}_{4}(u)(t, x)=\int_{0}^{t} \int_{\mathbb{R}^{d}} \mathbb{G}_{\alpha, \delta}(t-s, x-y) \sigma(s, y, u(s, y)) \\
& \mathscr{T}_{5}(u)(t, x)=\int_{0}^{t} \int_{\mathbb{R}^{d}} \int_{E_{2}} \mathbb{G}_{\alpha, \delta}(t-s, x-y) \sigma(s, y, u(s, y)) \\
& \times h(s, y, z) M(d z, d y, d s) .
\end{aligned}
$$

According to (43), we have the following.

Proposition 8. Suppose $1+\sum_{i=1}^{d}\left(1 / \alpha_{i}-1 / h \alpha_{i}\right)>0$ and the assumptions (1), (2), and (3) of Theorem 6 are satisfied. Then, for each $p>2\left(1+\sum_{i=1}^{d}\left(1 / \alpha_{i}\right)\right) / \sum_{i=1}^{d}\left(1 / \alpha_{i}\right)-1$ and $u \in \mathbb{B}$, it holds that $\mathscr{T} \in \mathbb{B}$.

Proof. From Lemma 3 and Young inequality, it follows that

$$
\begin{aligned}
& \left\|\mathcal{T}_{1}(u)(t, x)\right\|_{p} \\
& =\left\|\int_{\mathbb{R}^{d}} \mathbb{G}_{\alpha, \delta}(t, x-y) u_{0}(y) d y\right\|_{p} \\
& =t^{-\sum_{i=1}^{d}\left(1 / \alpha_{i}\right)}\left\|\int_{\mathbb{R}^{d}} \prod_{i=1}^{d} G_{\alpha_{i}, \delta_{i}}\left(1, t^{-1 / \alpha_{i}}\left(\cdot-y_{i}\right)\right) u_{0}(y) d y\right\|_{p} \\
& \leq t^{-\sum_{i=1}^{d}\left(1 / \alpha_{i}\right)}\left\|\left[\prod_{i=1}^{d} G_{\alpha_{i}, \delta_{i}}\left(1, t^{-1 / \alpha_{i}} \cdot\right) * u_{0}(\cdot)\right](\cdot)\right\|_{p} \\
& \leq t^{-\sum_{i=1}^{d}\left(1 / \alpha_{i}\right)}\left\|\prod_{i=1}^{d} G_{\alpha_{i}, \delta_{i}}\left(1, t^{-1 / \alpha_{i}} \cdot\right)\right\|_{1} \cdot\left\|u_{0}(\cdot)\right\|_{p} \\
& \leq C\left\|u_{0}(\cdot)\right\|_{p}<\infty,
\end{aligned}
$$

where we have used the fact that $\mathbb{E}\left[\left\|u_{0}(\cdot)\right\|_{p}^{p}\right]<\infty$. Now we deal with $\mathscr{T}_{2}(u)$. Applying (a) of Lemma 7 with $1 / r=1 / p-$ $1 / p+1=1$ and condition (1) of Theorem 6 , we conclude that

$$
\begin{aligned}
& \mathbb{E}\left[\left\|\mathcal{T}_{2}(u)(t, x)\right\|_{p}^{p}\right] \\
& \leq C \mathbb{E}\left[\int_{0}^{t}(t-s)^{\sum_{i=1}^{d}\left((r-1) / \alpha_{i}\right)}\|b(s, \cdot u(s, \cdot))\|_{p} d s\right]^{p}
\end{aligned}
$$




$$
\begin{aligned}
& \leq C \mathbb{E}\left[\int_{0}^{t}\left(1+\|u(s, \cdot)\|_{p}\right) d s\right]^{p} \\
& \leq C_{p, T}\left[1+\sup _{0 \leq t \leq T} \mathbb{E}\|u(s, \cdot)\|_{p}^{p}\right] \\
& \leq C_{p, T}\left[1+\|u(s, \cdot)\|_{\mathbb{B}}^{p}\right]<\infty,
\end{aligned}
$$

since $u \in \mathbb{B}$. that

Now, let us consider $\mathscr{T}_{3}(u)(t, x)$. By Lemma 3, we deduce

$$
\begin{aligned}
& \mathbb{E}\left\|\mathscr{T}_{3}(u)(t, x)\right\|_{p}^{p} \\
& =\int_{\mathbb{R}^{d}} \mathbb{E}\left|\int_{0}^{t} \int_{\mathbb{R}^{d}} \mathbb{G}_{\alpha, \delta}(t-s, x-y) B^{H}(d y, d s)\right|^{p} d x \\
& =\int_{\mathbb{R}^{d}} \mathbb{E}\left|\int_{0}^{t} \int_{\mathbb{R}^{d}}\left(K_{H}^{*} G_{\alpha, \delta}(t-\cdot, x-\cdot)\right)(s, y) W(d y, d s)\right|^{p} d x \\
& \leq C_{p} \int_{\mathbb{R}^{d}}\left\|\mathbb{G}_{\alpha, \delta}(t-\cdot, x-\cdot)\right\|_{L^{1 / h}\left([0, T] \times \mathbb{R}^{d}\right)}^{p} d x,
\end{aligned}
$$

where $W(s, y)$ is a space-time white noise on $[0, T] \times \mathbb{R}^{d}$.

Note that

$$
\begin{aligned}
& \left\|\mathbb{G}_{\alpha, \delta}(t-\cdot, x-*)\right\|_{L^{1 / h}\left([0, T] \times \mathbb{R}^{d}\right)}^{p} \\
& =\left[\int_{0}^{t} \int_{\mathbb{R}^{d}}\left|\mathbb{G}_{\alpha, \delta}(t-s, x-y)\right|^{1 / h} d y d s\right]^{p h} \\
& =\left[\int_{0}^{t}(t-s)^{-\sum_{i=1}^{d}\left(1 / h \alpha_{i}\right)}\right. \\
& \left.\quad \times \int_{\mathbb{R}^{d}}\left|\prod_{i=1}^{d} G_{\alpha_{i}, \delta_{i}}\left(1,(t-s)^{-1 / \alpha_{i}}\left(x_{i}-y_{i}\right)\right)\right|^{1 / h} d y d s\right]^{p h} \\
& =\left[\int_{0}^{t}(t-s)^{\sum_{i=1}^{d}\left(1 / \alpha_{i}-1 / h \alpha_{i}\right)}\right. \\
& \left.\quad \times \prod_{i=1}^{d}\left(\int_{\mathbb{R}}\left|G_{\alpha_{i}, \delta_{i}}\left(1, z_{i}\right)\right|^{1 / h} d z_{i}\right) d s\right]^{p h} \\
& \leq C_{\alpha, H}\left[\int_{0}^{t}(t-s)^{\sum_{i=1}^{d}\left(1 / \alpha_{i}-1 / h \alpha_{i}\right)} d s\right]^{p h}<\infty,
\end{aligned}
$$

under the assumption $1+\sum_{i=1}^{d}\left(1 / \alpha_{i}-1 / h \alpha_{i}\right)>0$. So we have $\mathscr{T}_{3}(u)(t, x) \in \mathbb{B}$ for $p \geq 2$.
Combining (a) of Lemma 7 with $1 / r=1 / p-2 / p+1=$ $1-1 / p \in(0,1]$ and assumption (33), we obtain that, for $p \in$ $[2, \infty)$,

$$
\begin{aligned}
& \mathbb{E}\left\|\mathscr{T}_{4}(u)(t, x)\right\|_{p}^{p} \\
& \leq C \mathbb{E}\left[\int_{0}^{t}(t-s)^{-\left(\sum_{i=1}^{d}\left(1 / \alpha_{i}\right)\right)(1-1 / r)}\|1+|u(s, \cdot)|\|_{p} \cdot\|\psi\|_{p} d s\right]^{p} \\
& \leq C_{p}\left[1+\|u(\cdot)\|_{\mathbb{B}}^{p}\right] \sup _{0 \leq t \leq T}\|\psi(t, \cdot)\|_{p}^{p} \\
& \cdot\left[\int_{0}^{t}(t-s)^{-\left(\sum_{i=1}^{d}\left(1 / \alpha_{i}\right)\right)(1-1 / r)(p /(p-1))} d s\right]^{p-1} \\
& \leq C_{p, T} \sup _{0 \leq t \leq T}\|\psi(t, \cdot)\|_{p}^{p}\left(1+\|u(\cdot)\|_{\mathbb{B}}^{p}\right) .
\end{aligned}
$$

with $1-\left(1 /\left(\sum_{i=1}^{d}\left(1 / \alpha_{i}\right)\right)(p-1)\right)>0$; that is, $p>1+$ $\left(1 / \sum_{i=1}^{d}\left(1 / \alpha_{i}\right)\right)$. Finally, let us estimate $\mathscr{T}_{5}(u)(t, x)$. This is a key step in the proof of Proposition 8. Together with the condition (34), (b) of Lemma 7 with $1 / r=1 / p-4 / p+1=$ $1-2 / p \in(0,1]$ and Lemma 4 , we conclude that, for $p>$ $2\left(1+\sum_{i=1}^{d}\left(1 / \alpha_{i}\right)\right) /\left(\sum_{i=1}^{d}\left(1 / \alpha_{i}\right)-1\right)$

$$
\begin{gathered}
\mathbb{E}\left\|\mathscr{T}_{5}(u)(t, \cdot)\right\|_{p}^{p} \\
=\mathbb{E}\left[\| \int_{0}^{t+} \int_{\mathbb{R}^{d}} \int_{E_{2}} \mathbb{G}_{\alpha, \delta}(t-s, \cdot-y) \sigma(s, y, u(s, y))\right. \\
\left.\times h(s, y, z) M(d y, d z, d s) \|_{p}\right] \\
\leq C_{p} \int_{\mathbb{R}^{d}}\left[\int_{0}^{t} \int_{\mathbb{R}^{d}} \int_{E_{2}}\left|\mathbb{G}_{\alpha, \delta}(t-s, x-y) h(s, y, z)\right|^{2}\right. \\
\times\left(\mathbb{E}\left[1+|u(s, y)|^{p}\right]\right)^{2 / p} \\
\left.\times C_{p} \| \int_{0}^{t} \int_{\mathbb{R}^{d}}\left|\mathbb{G}_{\alpha, \delta}(t-s, x-y)\right|^{2}(d z) d y d s\right]^{p / 2} d x \\
\times\left[\int_{E_{2}}|h(s, y, z)|^{2} \mu_{2}(d z)\right] \\
\times\left[1+\mathbb{E}|u(s, \cdot)|^{p}\right]^{2 / p} d y d s \|^{p / 2}
\end{gathered}
$$




$$
\begin{aligned}
& \leq C_{p}\left[\int_{0}^{t}(t-s)^{-(p+2) / p\left(\sum_{i=1}^{d}\left(1 / \alpha_{i}\right)\right)}\right. \\
& \times \|\left[\int_{E_{2}}|h(s, y, z)|^{2} \mu_{2}(d z)\right] \\
& \left.\times\left[1+\mathbb{E}|u(s, \cdot)|^{p}\right]^{2 / p} \|_{p / 4} d s\right]^{p / 2} \\
& \leq C_{p}\left[\int_{0}^{t}(t-s)^{-(p+2) / p\left(\sum_{i=1}^{d}\left(1 / \alpha_{i}\right)\right)}\right. \\
& \times\left\|\int_{E_{2}}|h(s, y, z)|^{2} \mu_{2}(d z)\right\|_{p / 2} \\
& \left.\times\left\|\left(1+\mathbb{E}|u(s, \cdot)|^{p}\right)^{2 / p}\right\|_{p / 2} d s\right]^{p / 2} \\
& \leq C_{p}\left[\sup _{0 \leq t \leq T}\left\|\int_{E_{2}}|h(s, y, z)|^{2} \mu_{2}(d z)\right\|_{p / 2}^{p / 2}\right. \\
& \left.\cdot \sup _{0 \leq t \leq T}\left\|\left(1+\mathbb{E}|u(s, \cdot)|^{p}\right)^{2 / p}\right\|_{p / 2}^{p / 2}\right] \\
& \cdot\left[\int_{0}^{t}(t-s)^{-(p+2) /(p-2)\left(\sum_{i=1}^{d}\left(1 / \alpha_{i}\right)\right)}\right]^{(p-2) / 2} \\
& \leq C_{p, T}\left[\sup _{0 \leq t \leq T}\left\|\int_{E_{2}}|h(s, y, z)|^{2} \mu_{2}(d z)\right\|_{p / 2}^{p / 2}\right] \\
& \times\left(1+\|u(\cdot)\|_{\mathbb{B}}^{p}\right)<\infty .
\end{aligned}
$$

Thus we have proved that the operator $\mathscr{T}$ defined by (43) is an operator from $\mathbb{B}$ to itself. On the other hand, from the similar argument as in (45)-(50), let $\eta>0$ sufficiently large; then $\mathscr{T} \in \mathbb{B}$. Thus we complete the proof of the proposition.

In what follows, we will prove that the operator $\mathscr{T}: \mathbb{B} \mapsto$ $\mathbb{B}$ is a contract operator.

Proposition 9. Suppose the assumptions (1), (2), and (3) of Theorem 6 are satisfied. Then, for each $p>2(1+$ $\left.\sum_{i=1}^{d}\left(1 / \alpha_{i}\right)\right) /\left(\sum_{i=1}^{d}\left(1 / \alpha_{i}\right)-1\right)$, the operator $\mathscr{T}_{\alpha}$ is a contraction on $\mathbb{B}$. In other words, there exists a constant $\varrho \in(0,1)$ such that

$$
\|\mathscr{T}(u)-\mathscr{T}(v)\|_{\mathbb{B}} \leq \varrho\|u-v\|_{\mathbb{B}}, \quad \text { for } u, v \in \mathbb{B} .
$$

Proof. Let $u_{0}$ and $v_{0}$ be initials of $\left(\mathscr{F}_{t}\right)_{t \geq 0}$-adapted random fields $u, v \in \mathbb{B}$ such that $u_{0}=v_{0}$. Let us consider $\mathscr{T}_{1}(u)$ firstly. Note that, for $\rho=p / 3$, together with (a) of Lemma 7 with $1 / r=(1 / p)-(1 / \rho)+1=1-(2 / p)$ and condition (1) of Theorem 6 , one can get

$$
\begin{aligned}
& \mathbb{E}\left[\left\|\mathscr{T}_{2}(u)(t, \cdot)-\mathscr{T}_{2}(v)(t, \cdot)\right\|_{p}^{p}\right] \\
& \leq C \mathbb{E}\left[\int_{0}^{t}(t-s)^{\sum_{i=1}^{d}\left((r-1) / \alpha_{i}\right)}\right. \\
& \left.\quad \times\|b(s, y, u(s, y))-b(s, y, v(s, y))\|_{p} d s\right]^{p} \\
& \leq C_{p}\left[\int_{0}^{t}(t-s)^{\sum_{i=1}^{d}\left((r-1) / \alpha_{i}\right)} \mathbb{E}\|u(s, \cdot)-v(s, \cdot)\|_{p} d s\right]^{p}
\end{aligned}
$$

Then

$$
\begin{aligned}
& \left\|\mathscr{T}_{2}(u)(t, \cdot)-\mathscr{T}_{2}(v)(t, \cdot)\right\|_{\mathbb{B}}^{p} \\
& =\sup _{0 \leq t \leq T} e^{-\eta t} \mathbb{E}\left[\left\|\mathscr{T}_{2}(u)(t, \cdot)-\mathscr{T}_{2}(v)(t, \cdot)\right\|_{p}^{p}\right] \\
& \leq C \sup _{0 \leq t \leq T} \mathbb{E}\left[\int_{0}^{t} e^{-(\eta / p)(t-s)}(t-s)^{\sum_{i=1}^{d}\left((r-1) / \alpha_{i}\right)} e^{-(\eta / p) s}\right. \\
& \left.\quad \times\|u(s, \cdot)-v(s, \cdot)\|_{p} d s\right]^{p} \\
& \leq C_{p} \sup _{0 \leq t \leq T} \mathbb{E}\left[\int_{0}^{t} e^{-\eta s}\|u(s, \cdot)-v(s, \cdot)\|_{p}^{p} d s\right] \\
& \quad \cdot\left[\int_{0}^{t}\left(e^{-(\eta / p)(t-s)}(t-s)^{\sum_{i=1}^{d}\left((r-1) / \alpha_{i}\right)}\right)^{p /(p-1)} d s\right]^{p-1} \\
& \leq C_{p} \sup _{0 \leq t \leq T}\left[\int_{0}^{t} e^{-\eta s} \mathbb{E}\|u(s, \cdot)-v(s, \cdot)\|_{p}^{p} d s\right] \\
& \quad\left[\int_{0}^{t}\left(e^{-(\eta / p)(t-s)}(t-s)^{-(1 / \alpha)(1-r)}\right)^{p /(p-1)} d s\right]^{p-1} \\
& \leq \varrho\|u-v\|_{\mathbb{B}}^{p},
\end{aligned}
$$

with $\varrho \in(0,1)$ by choosing $\eta>0$ large enough. Now we are going to consider the term $\mathscr{T}_{5}(u)(t, x)$. From a similar argument as in (50), thanks to the generalized B-D-G inequality, we derive from the conditions of Theorem 6 that

$$
\begin{aligned}
& \left\|\mathscr{T}_{5}(u)(t, \cdot)-\mathscr{T}_{5}(v)(t, \cdot)\right\|_{\mathbb{B}}^{p} \\
& \quad=\sup _{0 \leq t \leq T} e^{-\eta t} \mathbb{E}\left[\left\|\mathscr{T}_{5}(u)(t, \cdot)-\mathscr{T}_{5}(v)(t, \cdot)\right\|_{p}^{p}\right]
\end{aligned}
$$




$$
\begin{gathered}
\sup _{0 \leq t \leq T} e^{-\eta t} \int_{\mathbb{R}^{d}} \mathbb{E} \mid \int_{0}^{t+} \int_{\mathbb{R}^{d}} \int_{E_{2}} \mathbb{G}_{\alpha, \delta}(t-s, x-y) h(s, y, z) \\
\cdot[\sigma(s, y, u(s, y)) \\
-\sigma(s, y, v(s, y))] \\
\times\left. M(d y, d z, d s)\right|^{p} d x
\end{gathered}
$$$$
\leq C_{p} \sup _{0 \leq t \leq T} e^{-\eta t}
$$$$
\times \int_{\mathbb{R}^{d}}\left(\int_{0}^{t} \int_{\mathbb{R}^{d}} \int_{E_{2}}\left|\mathbb{G}_{\alpha, \delta}(t-s, x-y) h(s, y, z)\right|^{2}\right.
$$$$
\cdot\left(\mathbb{E}|u(s, y)-v(s, y)|^{p}\right)^{2 / p}
$$$$
\left.\times \mu_{2}(d z) d y d s\right)^{p / 2} d x
$$

$$
\begin{gathered}
\leq C_{p} \sup _{0 \leq t \leq T} e^{-\eta t} \\
\times \int_{\mathbb{R}^{d}}\left(\int_{0}^{t} \int_{\mathbb{R}^{d}} \int_{E_{2}}\left|\mathbb{G}_{\alpha, \delta}(t-s, x-y) h(s, y, z)\right|^{2} e^{2 \eta s / p}\right. \\
\cdot\left(e^{-\eta s} \mathbb{E}|u(s, y)-v(s, y)|^{p}\right)^{2 / p} \\
\left.\times \mu_{2}(d z) d y d s\right)^{p / 2} d x
\end{gathered}
$$

$$
\begin{aligned}
& \leq C_{p} \sup _{0 \leq t \leq T} \\
& \quad \times \int_{\mathbb{R}^{d}}\left(\int_{0}^{t} \int_{\mathbb{R}^{d}} \int_{E_{2}} e^{-\eta s} \mathbb{E}|u(s, y)-v(s, y)|^{p} \mu_{2}(d z) d y d s\right) \\
& \cdot\left(\int_{0}^{t} \int_{\mathbb{R}^{d}} \int_{E_{2}}\left|\mathbb{G}_{\alpha, \delta}(t-s, x-y) h(s, y, z)\right|^{2 p /(p-2)}\right. \\
& \left.\times C_{p, T} \mu_{2}\left(E_{2}\right)\|u-v\|_{\mathbb{B}}^{p} e^{-(2 \eta(t-s) /(p-2))} \mu_{2}(d z) d y d s\right)^{(p-2) / 2} d x \\
& \quad \sup _{0 \leq t \leq T} \int_{\mathbb{R}^{d}} \int_{0}^{t} \int_{\mathbb{R}^{d}} \int_{E_{2}}\left|\mathbb{G}_{\alpha, \delta}(t-s, x-y) h(s, y, z)\right|^{p} \\
& \times e^{-\eta(t-s)} \mu_{2}(d z) d y d s d x
\end{aligned}
$$$$
\leq \varrho\|u-v\|_{\mathbb{B}}^{p}
$$

Then $\mathscr{T}_{5}(u)$ is a contraction on $\mathbb{B}$.

A similar procedure as the above arguments yields that $\mathscr{T}_{4}(u)$ is a contraction on $\mathbb{B}$ by letting $\eta>0$ large enough. Therefore, it follows from (49) that $\mathscr{T}(\cdot)$ is a contraction on $\mathbb{B}$ if $\eta>0$ large enough. Thus the proof of Proposition 9 is complete.
Based on Propositions 8 and 9 and fixed point principal on the set $\left\{u \in \mathbb{B}: u(0)=u_{0}\right\}$, we conclude that (3) admits a unique solution $u \in \mathbb{B}$. Thus the conclusion of Theorem 6 follows.

\section{Conflict of Interests}

The authors declare that there is no conflict of interests regarding the publication of this paper.

\section{Acknowledgments}

The authors would like to express their sincere gratitude to the editor and the anonymous referees for their valuable comments and error corrections. Xichao Sun is partially supported by Natural Science Foundation of Anhui Province (no. 1408085QA10), Key Natural Science Foundation of Anhui Education Commission (no. KJ2013A183), and Mathematical Tianyuan Foundation of China (no. 11226198). Zhi Wang is partially supported by the Research Project of Education of Zhejiang Province (Y201326507) and the Natural Science Foundation of Ningbo Municipality (201401A6111010). Jing Cui is partially supported by the National Natural Science Foundation of China (11326171), the Natural Science Foundation of Anhui Province (1308085QA14), the Key Natural Science Foundation of Anhui Educational Committee (KJ2013A133), and the PhD Start-up Fund of Anhui Normal University.

\section{References}

[1] J. B. Walsh, "An introduction to stochastic partial differential equations," in École d'été de Probabilités de Saint-Flour, XIV1984, vol. 1180 of Lecture Notes in Math., pp. 265-439, Springer, Berlin, Germany, 1986.

[2] L. Debbi and M. Dozzi, "On the solutions of nonlinear stochastic fractional partial differential equations in one spatial dimension," Stochastic Processes and Their Applications, vol. 115, no. 11, pp. 1764-1781, 2005.

[3] L. Boulanba, M. Eddahbi, and M. Mellouk, "Fractional SPDEs driven by spatially correlated noise: existence of the solution and smoothness of its density," Osaka Journal of Mathematics, vol. 47, no. 1, pp. 41-65, 2010.

[4] C. Mueller, "The heat equation with Lévy noise," Stochastic Processes and Their Applications, vol. 74, no. 1, pp. 67-82, 1998.

[5] J. Wu, "Fractal Burgers equation with stable Lévy noise," in International Conference SPDE and Applications-VII, January 2004

[6] T. Chang and K. Lee, "On a stochastic partial differential equation with a fractional Laplacian operator," Stochastic Processes and Their Applications, vol. 122, no. 9, pp. 3288-3311, 2012.

[7] A. Truman and J. Wu, "Fractal Burgers equation driven by Lévy noises," in Stochastic Partial Differential Equations and Applications, vol. 245 of Lect. Notes Pure and Appl. Math., pp. 295-310, Chapman \& Hall/CRC Taylor \& Francis, Boca Raton, Fla, USA, 2006.

[8] J. Liu, L. Yan, and Y. Cang, "On a jump-type stochastic fractional partial differential equation with fractional noises," Nonlinear Analysis: Theory, Methods \& Applications, vol. 75, no. 16, pp. 6060-6070, 2012. 
[9] $\mathrm{D} . \mathrm{Wu}$, "On the solution process for a stochastic fractional partial differential equation driven by space-time white noise," Statistics \& Probability Letters, vol. 81, no. 8, pp. 1161-1172, 2011.

[10] Y. Hu, "Heat equations with fractional white noise potentials," Applied Mathematics and Optimization, vol. 43, no. 3, pp. 221243, 2001.

[11] T. T. Wei, "The high-order SPDEs driven by multi-parameter fractional noises," Acta Mathematica Sinica, vol. 26, no. 10, pp. 1943-1960, 2010.

[12] Y. Hu and D. Nualart, "Stochastic heat equation driven by fractional noise and local time," Probability Theory and Related Fields, vol. 143, no. 1-2, pp. 285-328, 2009.

[13] R. M. Balan and C. A. Tudor, "Stochastic heat equation with multiplicative fractional-colored noise," Journal of Theoretical Probability, vol. 23, no. 3, pp. 834-870, 2010.

[14] R. M. Balan and C. A. Tudor, "The stochastic wave equation with fractional noise: a random field approach," Stochastic Processes and Their Applications, vol. 120, no. 12, pp. 2468-2494, 2010.

[15] L. Bo, Y. Jiang, and Y. Wang, "On a class of stochastic Anderson models with fractional noises," Stochastic Analysis and Applications, vol. 26, no. 2, pp. 256-273, 2008.

[16] L. Bo, Y. Jiang, and Y. Wang, "Stochastic Cahn-Hilliard equation with fractional noise," Stochastics and Dynamics, vol. 8, no. 4, pp. 643-665, 2008.

[17] Y. Jiang, K. Shi, and Y. Wang, "Stochastic fractional Anderson models with fractional noises," Chinese Annals of Mathematics. Series B, vol. 31, no. 1, pp. 101-118, 2010.

[18] Y. Jiang, X. Wang, and Y. Wang, "On a stochastic heat equation with first order fractional noises and applications to finance," Journal of Mathematical Analysis and Applications, vol. 396, no. 2, pp. 656-669, 2012.

[19] Y. Jiang, T. Wei, and X. Zhou, "Stochastic generalized Burgers equations driven by fractional noises," Journal of Differential Equations, vol. 252, no. 2, pp. 1934-1961, 2012.

[20] K. Shi and Y. Wang, "On a stochastic fractional partial differential equation with a fractional noise," Stochastics, vol. 84, no. 1 , pp. 21-36, 2012.

[21] S. Albeverio, J.-L. Wu, and T.-S. Zhang, "Parabolic SPDEs driven by Poisson white noise," Stochastic Processes and Their Applications, vol. 74, no. 1, pp. 21-36, 1998.

[22] K. Shi and Y. Wang, "On a stochastic fractional partial differential equation driven by a Lévy space-time white noise," Journal of Mathematical Analysis and Applications, vol. 364, no. 1, pp. 119-129, 2010.

[23] A. Truman and J. Wu, "Stochastic Burgers equation with Lévy space-time white noises," in Probabilistic Methods in Fluids, pp. 298-323, World Scientific, Singapore, 2003.

[24] A. Truman and J. Wu, Fractal Burgers Equation Driven by Lévy Noises, vol. 245, of Lecture Notes in Pure and Applied Mathematics, Chapman \& Hall/CRC Taylor \& Francis, Boca Raton, Fla, USA, 2006.

[25] J.-L. Wu and B. Xie, "On a Burgers type nonlinear equation perturbed by a pure jump Lévy noise in $\mathbb{R}^{d}$," Bulletin des Sciences Mathématiques, vol. 136, no. 5, pp. 484-506, 2012.

[26] A. Løkka, B. Øksendal, and F. Proske, "Stochastic partial differential equations driven by Lévy space-time white noise," The Annals of Applied Probability, vol. 14, no. 3, pp. 1506-1528, 2004.

[27] L. Bo and Y. Wang, "Stochastic Cahn-Hilliard partial differential equations with Lévy spacetime white noises," Stochastics and Dynamics, vol. 6, no. 2, pp. 229-244, 2006.
[28] J. Mémin, Y. Mishura, and E. Valkeila, "Inequalities for the moments of Wiener integrals with respect to a fractional Brownian motion," Statistics \& Probability Letters, vol. 51, no. 2, pp. 197-206, 2001.

[29] L. Debbi, "On some properties of a high order fractional differential operator which is not in general selfadjoint," Applied Mathematical Sciences, vol. 1, no. 25-28, pp. 1325-1339, 2007.

[30] T. Komatsu, "On the martingale problem for generators of stable processes with perturbations," Osaka Journal of Mathematics, vol. 21, no. 1, pp. 113-132, 1984. 


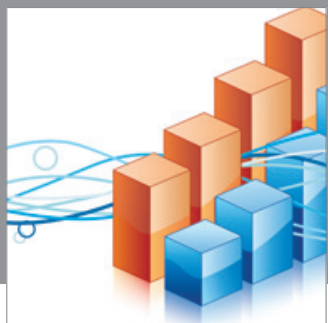

Advances in

Operations Research

mansans

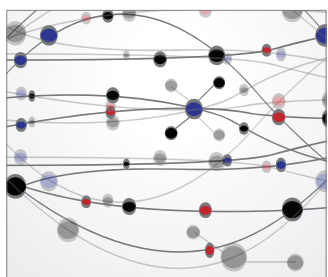

The Scientific World Journal
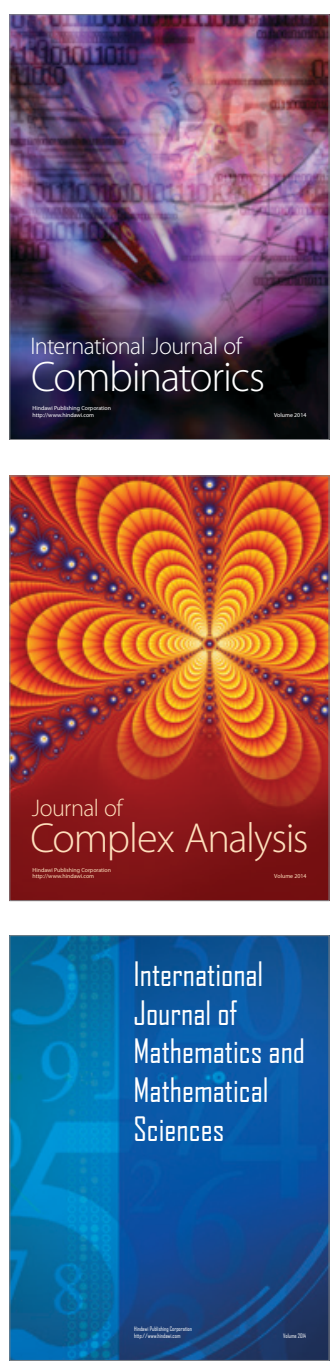
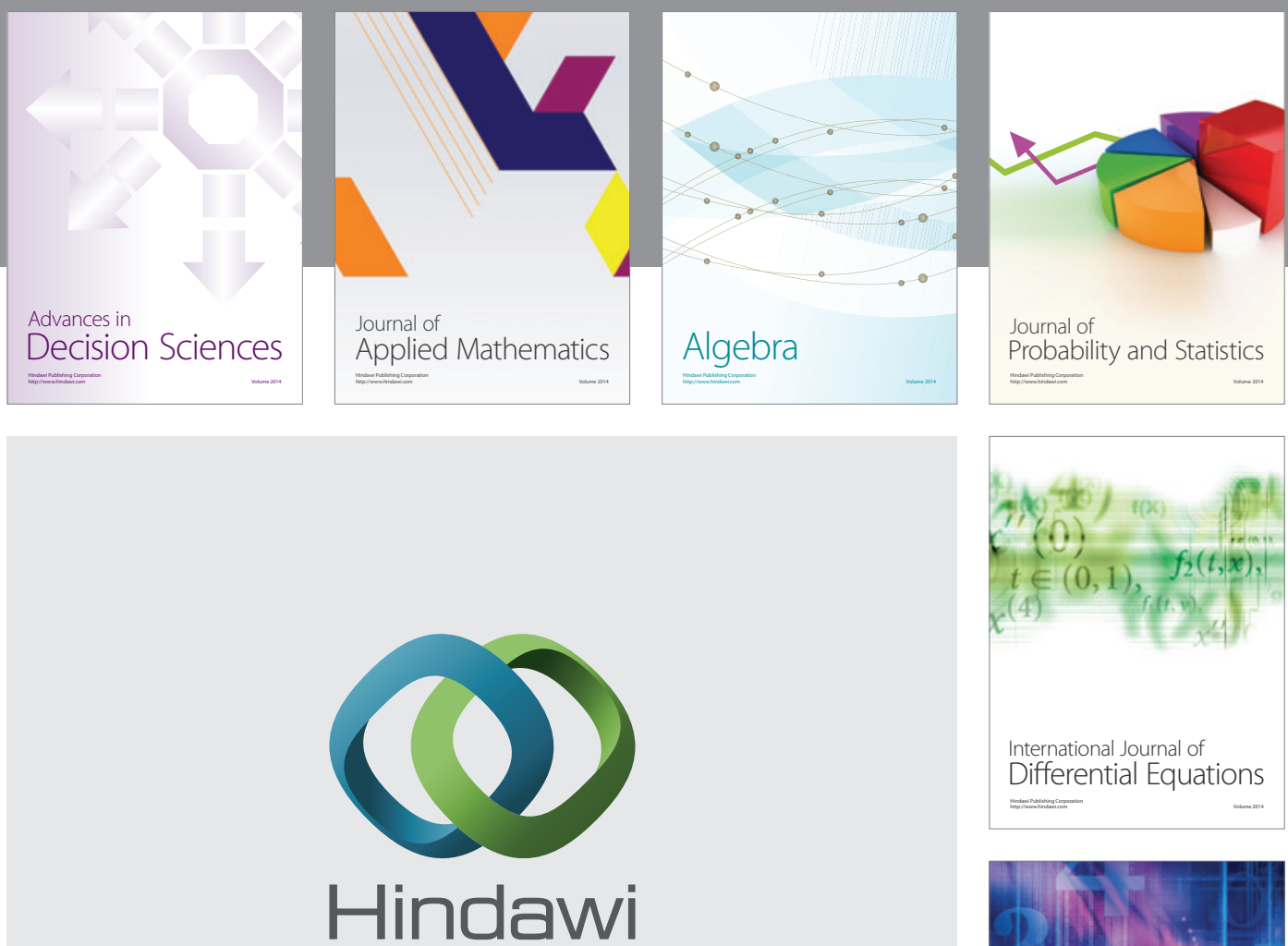

Submit your manuscripts at http://www.hindawi.com
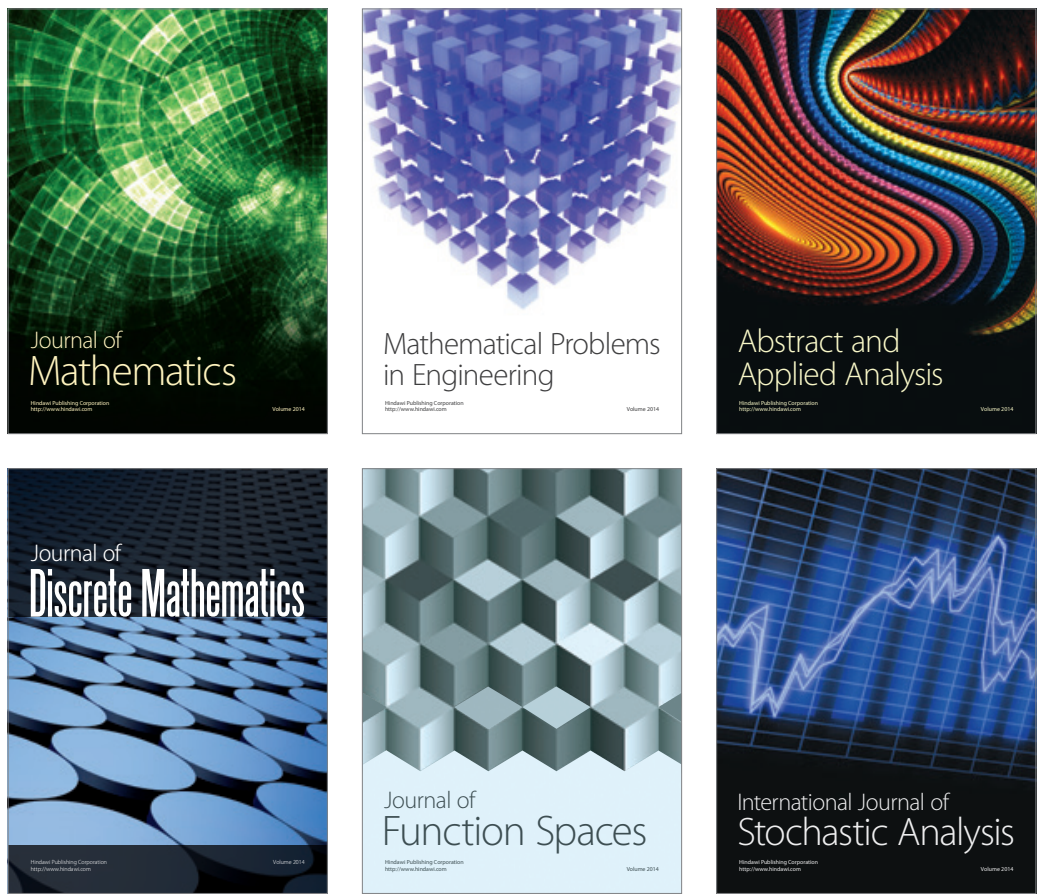

Journal of

Function Spaces

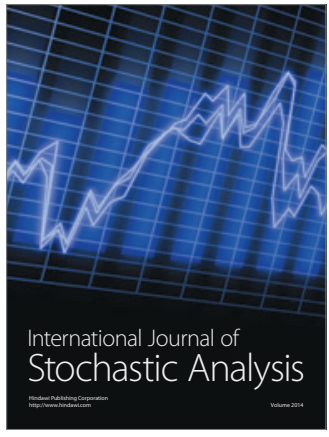

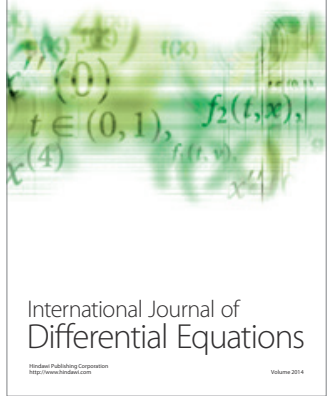
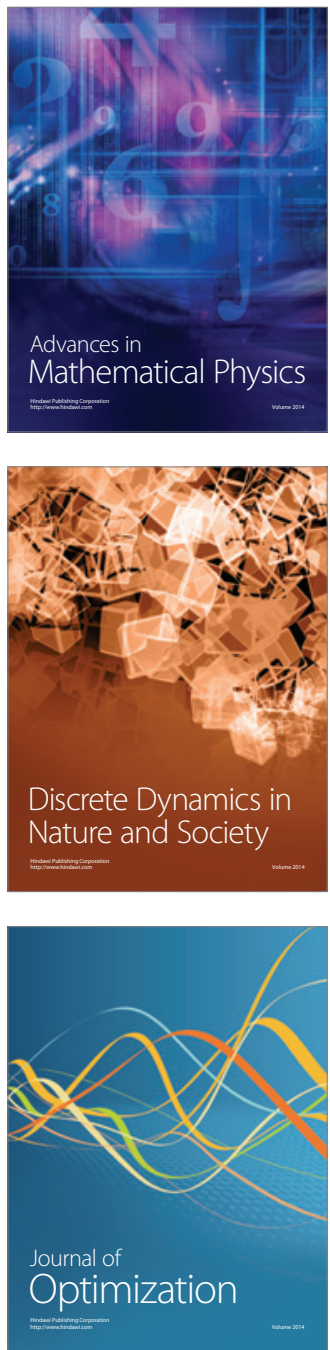